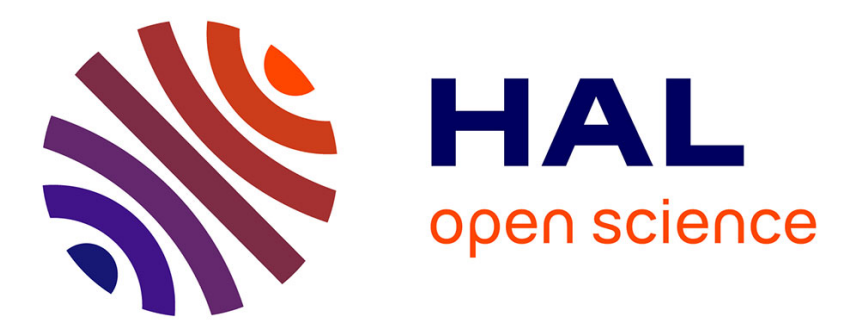

\title{
On the consensus of heterogeneous multi-agent systems: a decoupling approach
}

\author{
Gabriel Rodrigues de Campos, Lara Briñon Arranz, Alexandre Seuret, \\ Silviu-Iulian Niculescu
}

\section{- To cite this version:}

Gabriel Rodrigues de Campos, Lara Briñon Arranz, Alexandre Seuret, Silviu-Iulian Niculescu. On the consensus of heterogeneous multi-agent systems: a decoupling approach. NecSys 2012 - 3rd IFAC Workshop on Distributed Estimation and Control in Networked Systems, Sep 2012, Santa-Barbara, United States. pp.1-6. hal-00726264

\section{HAL Id: hal-00726264 https://hal.science/hal-00726264}

Submitted on 29 Aug 2012

HAL is a multi-disciplinary open access archive for the deposit and dissemination of scientific research documents, whether they are published or not. The documents may come from teaching and research institutions in France or abroad, or from public or private research centers.
L'archive ouverte pluridisciplinaire HAL, est destinée au dépôt et à la diffusion de documents scientifiques de niveau recherche, publiés ou non, émanant des établissements d'enseignement et de recherche français ou étrangers, des laboratoires publics ou privés. 


\title{
On the Consensus of Heterogeneous Multi-Agent Systems: a Decoupling Approach
}

\author{
Gabriel Rodrigues de Campos* Lara Briñón-Arranz* \\ Alexandre Seuret* Silviu-Iulian Niculescu ${ }^{* *}$ \\ * NeCS Team, Automatic Control Department of GIPSA-Lab, \\ Grenoble, France \\ ** Laboratory of Signals and Systems (L2S) \\ CNRS-Supelec, Gif-sur-Yvette, France
}

\begin{abstract}
This article deals with consensus algorithms for heterogeneous multi-agent systems (MAS). A control strategy based on a consensus algorithm which is decoupled from the original systems is proposed. Consequently, its major advantage remains in the separation of the stability analysis of each subsystem and the distributed control algorithm. Through the paper, it is shown that our method allows using classical distributed consensus algorithms such as simple integrator consensus (with or without delay) and distributed consensus filter algorithms. Finally, some simulations supporting our theoretical results are presented, where the efficiency of the method is tested for completely arbitrary models.
\end{abstract}

Keywords: Consensus, Heterogeneous agents, Multi-agent systems, Delay systems

\section{INTRODUCTION}

Roughly speaking, Networked Control Systems (NCS) are spatially distributed systems with a communication network used between sensors, actuators, and controllers, which allows flexible architectures with reduced installation and maintenance costs. This means NCS's applications can be found in a large range of areas such as mobile sensor networks (Ögren et al. (2004)), remote surgery, haptic collaboration over Internet, multi-robot systems (Olfati-Saber and Murray (2004)), automated highway systems, averaging in communication networks (Xiao and Boyd (2004)) and formation control (Dimarogonas and Kyriakopoulos (2008); Ren (2007)). A recent review of the vast literature in the field can be found in Olfati-Saber et al. (2007), Hespanha et al. (2007) and Ren et al. (2005).

We consider a consensus algorithm (or protocol) as an interaction rule that specifies the information exchange between an agent and all of its neighbors over the network in order to reach an agreement regarding a certain quantity of interest that depends on the state of all agents, see, e.g., Ren and Beard (2007). Consensus algorithms are extensively studied in the literature for identical multi-agent systems and in particular to simple and double integrator dynamics, see, e.g., Olfati-Saber and Murray (2004); Ren and Beard (2007) and the references therein. However, increasing interest has turned to MAS with general linear time-invariant dynamical agents, see, e.g., Scardovi and Sepulchre (2009); Wieland et al. (2011a).

In this paper, we are interested in consensus algorithms for heterogeneous multi-agent systems, i.e., with non-identical dynamics representing, for example, different models or generations of robots. Assuming heterogeneous dynamics naturally fits to several challenging applications relying, for instance, on surface and underwater marine vehicles or on ground and aerial robots. Therefore, this work is motivated by recognition, recovery and search operations on a civil or military framework. For a system consisting of heterogeneous dynamical agents, the first question to be answered is whether there exists a consensus solution to such a system. Only a few papers consider heterogeneous cases of the synchronization problem. In particular, Chopra and Spong (2008); Qu et al. (2007) solved the output synchronization problem under a non linear approach. Recent results restrict their attention to heterogeneous linear dynamical systems Wieland et al. (2011b); Zheng et al. (2011); Kim et al. (2011). Consensus for heterogeneous multi-agent systems composed of simple and double integrators is presented in Zheng et al. (2011) while Jönsson and Kao (2010) pertains in consensus algorithms applied to a formation control problem. Moreover, the authors of Wieland et al. (2011b) focus on linear output synchronization of heterogeneous agents using an internal model approach. The same problem is analyzed in Kim et al. (2011) taking into account uncertainties of the agents' models.

This article proposes a control strategy based on a consensus algorithm which is decoupled from the original systems. In other words, we attribute to each agent an additional control variable which achieves a consensus and thus, the measurement variable of the each agent should converge to this additional variable. The new algorithm offers the major advantage to separate the stability analysis of each agent and the convergence analysis of the distributed consensus algorithm. To the best of the authors' knowledge, such approach is new and has not been reported in the literature.

This paper is organized as follows: Section 2 presents the problem formulation, while Section 3 will be devoted to the control design. In Section 4, we will proceed to stability analysis of the algorithm. Section 5 briefly presents extensions of the proposed algorithm to the case where delays appears in the communication network and to distributed consensus filters. Section 6 includes some illustrating simulation results. Finally, Section 7 will present our conclusions and indicate possible future research efforts. 
Notation: Throughout the paper, the superscript ' $T$ ' stands for matrix transposition, $\mathbb{R}^{n}$ denote the $n$ - dimensional Euclidean space, and $\mathbb{R}^{n \times m}$ is the set of $n \times m$ real matrices; $I_{m}$ represents the identity matrix of dimension $m \times m$. Finally, for any square matrix $M$, the notation $(M)_{i}$ denotes the $i^{t h}$ line of $M$ and $\lambda_{k}(M)$ represents the $k^{t h}$ eigenvalue of $M$. We denote for any $i \in \mathscr{N}$ $\mathscr{N}_{i}(t)$ as the subset of $\mathscr{N}$ including all neighbors of agent $i$, i.e, all nodes that agent $i$ can sense at time $t$. For the graph $G$ with $N$ vertices and an edge set given by $E=\left\{(i, j): j \in \mathscr{N}_{i}\right\}$ the adjacency matrix $\mathscr{A}=\mathscr{A}(G)=\left(a_{i j}\right)$ is a $N \times N$ matrix given by $a_{i j}=1$, if $(i, j) \in E$ and $a_{i j}=0$, otherwise. If there is an edge connecting two vertices $i, j$, i.e. $(i, j) \in E$, then $i, j$ are called adjacent. The degree $d_{i}$ of vertex $i$ is defined as the number of its neighboring vertices, i.e. $d_{i}=\# j:(i, j) \in E$. Denote also $d_{\text {max }}=\max \left\{d_{i}\right\}$. Let $\Delta$ be the $N \times N$ diagonal matrix of $d_{i}$ 's. The Laplacian of $G$ is the matrix $L=\Delta-\mathscr{A}$.

\section{PROBLEM STATEMENT}

In this section, we are going to study consensus algorithms for a set of heterogeneous linear systems. One can easily conclude that the control complexity for systems considering heterogeneous agents increases with respect to simpler frameworks. Even though some results for such problem have been proposed in literature, most of them presents major drawbacks such as computational effort, complexity or accuracy of the solution. Therefore, in this article an efficient way to simplify the control design is proposed.

\subsection{System Definition}

Consider the multi-agent linear system:

$$
\left\{\begin{array}{l}
\dot{x}_{i}=\bar{A}_{i} x_{i}+B_{i} u_{i} \\
y_{i}=x_{i} \\
z_{i}=C_{i} x_{i}
\end{array} \quad \forall i \in \mathscr{N}=\{1, \ldots, N\},\right.
$$

where $x_{i} \in \mathbb{R}^{n_{i}}, y_{i} \in \mathbb{R}^{n_{i}}, z_{i} \in \mathbb{R}^{m}$ and $u_{i} \in \mathbb{R}^{m}$ are the state, output, measurement and input vectors, respectively. Note that it is assumed that the state of the system is available for the design of the controller, i.e. $y_{i}=x_{i}$ for all $i \in \mathscr{N}$. For all $i \in \mathscr{N}$, the matrices $\bar{A}_{i} \in \mathbb{R}^{n_{i} \times n_{i}}, B_{i} \in \mathbb{R}^{n_{i} \times m}$ and $C_{i} \in \mathbb{R}^{m \times n_{i}}$, with $m<\min \left\{n_{i}\right\}$ and $n_{i}>m$ are assumed to be constant and known.

In this context, the objective of the paper is the design a distributed control law which ensures that (i) each subsystem is stable and (ii) the measurement vectors of each agent reach an agreement.

\subsection{Assumptions on the multi-agent system}

In order to solve this problem, the following assumptions on the systems are considered:

Assumption 1. (Heterogeneity): The $N$ systems are assumed to be heterogeneous.

In other words, this simply means that the matrices defining the systems may differ from one agent to another one and that the vectors $x_{i}$ may have different dimensions.

Assumption 2. (Homogeneity of the measurement vector): The measurement vectors $z_{i}$ represents the same quantity of interests for all agents. As a consequence, the measurement vectors have the same dimension, or in other words, $z_{i} \in$ $\mathbb{R}^{m} \forall i \in \mathscr{N}$, where $m<\min \left\{n_{i}\right\}$.

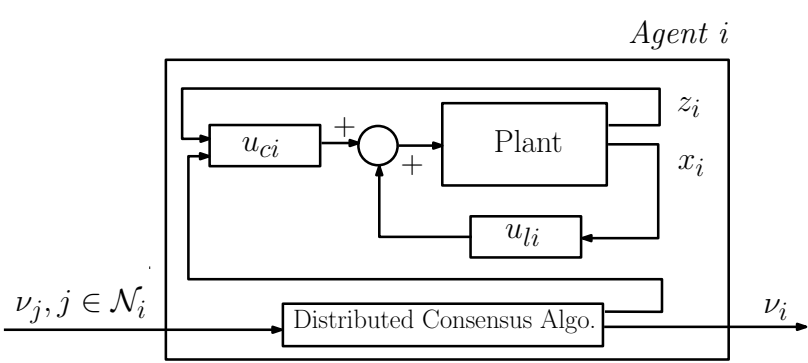

Fig. 1. Control architecture for agent $i$

Assumption 3. (Structures of the systems): For all $i \in \mathscr{N}$, the condition $\operatorname{rank}\left(C_{i} B_{i}\right)=m$ holds.

Technically speaking, it means that the input vectors directly affects the measurement vector.

Assumption 4. (Controllability): For each agent, the pair $\left(A_{i}, B_{i}\right)$ is controllable.

In order to avoid a centralized solution of the problem, the agents are assumed to be connected through a communication network. In order to establish further stability results, the following assumption holds.

Assumption 5. (Graph's connectivity): For any considered graph $G$, we assume that the communication graph has a directed spanning tree.

\section{CONTROLLER DESIGN}

In order to achieve the goals mentioned above, a controller composed of two parts, one corresponding to the local controller and one representing the consensus algorithm, is proposed. Thus, the control law for each agent is represented by:

$$
u_{i}(t)=u_{l i}(t)+u_{c i}(t), \quad i \in \mathscr{N},
$$

where $u_{l i}$ and $u_{c i}$ are the local and the agreement controllers respectively. The solution provided in this paper to solve this problem is summarized in Figure 1. In the sequel, a method is proposed for the design of the local and the agreement control laws.

\subsection{Local control law}

According to Assumption 4, for each system, there exists a local state feedback controller:

$$
u_{l i}=-K_{i} x_{i},
$$

such that the matrix $A_{i}=\bar{A}_{i}-B_{i} K_{i}$ is Hurwitz. Thus, dynamics (1) can the be written as:

$$
\left\{\begin{array}{l}
\dot{x}_{i}=A_{i} x_{i}+B_{i} u_{c i}, \\
z_{i}=C_{i} x_{i},
\end{array} \quad \forall i \in \mathscr{N}=\{1, \ldots, N\} .\right.
$$

The objective is to design a consensus algorithm which guarantees that the measurement vectors reach an agreement. In the literature, classical distributed consensus algorithms have been intensively studied. Inherently, their stability and performance properties are well documented (see, for instance, Olfati-Saber et al. (2007); Ren and Beard (2007); Hespanha et al. (2007) and the references therein).

\subsection{Distributed Consensus algorithm}

In the context of the paper, the main idea is to add additional dynamics to the control law of each system which correspond 
to a simple consensus algorithm. As a starting point, we will consider the simplest situation where the dynamics of this additional dynamics are driven by:

$$
\dot{v}_{i}=-\sum_{j \in \mathscr{N}_{i}}\left(v_{i}-v_{j}\right), \quad \forall i \in \mathscr{N},
$$

where $v_{i} \in \mathbb{R}^{m}$. This allows defining the augmented vector $v=$ $\left[v_{1}, \ldots, v_{N}\right]^{T}$ and thus the previous consensus algorithm can be rewritten in a matrix form such as $\dot{v}=-L \otimes I_{m} v$, where $L$ is the Laplacian matrix associated to the communication graph of the the multi agent system and $\otimes$ represents the classical Kronecker product.

The stability of such system has been widely studied in the literature, see, for example, Olfati-Saber et al. (2007); Ren and Beard (2007) among many others. The well known convergence properties of (5) naturally motivate it as an appropriated "choice" for the additional dynamics. The rest of the contribution consists in using this well known consensus algorithm to reach an agreement on those additional dynamics, while applying a standard model tracking based controller to the remaining system. This ensures that the real system will have identical performances as the additional model. Due to the interactions between them, $u_{c i}$ must be designed in a proper manner such that two correlated objectives are fulfilled:

$$
\left\{\begin{array}{l}
\lim _{t \rightarrow \infty}\left(v_{i}-v_{j}\right)=0, \\
\lim _{t \rightarrow \infty}\left(z_{i}-v_{i}\right)=0,
\end{array} \forall i, j \in \mathscr{N}^{2} .\right.
$$

In other words, system (5) can then be seen as a reference model for system (1). Then, it is natural to introduce the error vector between the measurement vector $z$ from (1) and the additional dynamics $v$ given by:

$$
\varepsilon_{i}=z_{i}-v_{i}
$$

We wish to e ensure that each $\varepsilon_{i}$ converges to zero as the time evolves, therefore the evolution of $\varepsilon_{i}$ has the following dynamics $\dot{\varepsilon}_{i}=-\beta \varepsilon_{i}$ where $\beta>0$. Thus, it follows:

$$
\begin{gathered}
\dot{z}_{i}-\dot{v}_{i}=-\beta\left(z_{i}-v_{i}\right), \\
C_{i}\left(A_{i} x_{i}+B_{i} u_{c i}\right)+(L)_{i} \otimes I_{m} v=-\beta \varepsilon .
\end{gathered}
$$

Due to the Assumption $3, C_{i} B_{i}$ is invertible for all agent $i$, and thus our controller can be expressed as a standard asymptotic output tracking controller (see Isidori (1995)) defined by:

$$
u_{c i}=\left(C_{i} B_{i}\right)^{-1}\left(\dot{v}_{i}-\beta\left(z_{i}-v_{i}\right)-C_{i} A_{i} x_{i}\right) \text {. }
$$

Remark 1. One might see that in the previous calculus we inherently assume that, at each time instant, each agent $i$ has access to its own full state. Despite the fact that, theoretical speaking, this does not represent a too conservative condition, the same comment does not hold in terms of practical application. However, this assumption might be relaxed by applying a observer and using the estimated state instead. Tough, for sake of brevity, the synthesis of this observer will not be considered.

Relying on a decoupling approach as well, a solution to the output synchronization problem for heterogeneous agents is presented in Wieland et al. (2011b). In particular, authors showed that an internal model requirement is necessary and sufficient for exponential synchronizability of a group of heterogeneous agents. Following Scardovi and Sepulchre (2009), the authors add an internal model to the dynamics of each agent and synchronize these identical exosystems in order to achieve output synchronization of the multi-agent systems.
Our objective is to obtain a very simple control technique to reach consensus of heterogeneous multi-agent systems. The main difference with respect to Wieland et al. (2011b) is that the heterogeneous synchronization problem is solved using the properties of simple-integrator consensus algorithms. Consequently, this approach can easily be extended to more complex and realistic situations where, for instance, communication delays or external references are considered. Moreover, this structure seems to considerably reduce the control complexity and computational efforts with respect to Wieland et al. (2011b).

\section{STABILITY ANALYSIS}

The next theorem states our main result:

Theorem 1. If Assumptions 1-5 are satisfied, then the control law (2), given by:

$$
u_{i}=-K_{i} x_{i}+\left(C_{i} B_{i}\right)^{-1}\left(\dot{v}_{i}-\beta\left(z_{i}-v_{i}\right)-C_{i} A_{i} x_{i}\right)
$$

where $\dot{v}_{i}$ is given by (5), guarantees that the multi-agent system (1) is stable and reaches an asymptotic measurement variable consensus.

\section{Proof.}

According to Assumption 3 and following Edwards and Spurgeon $(1995,1998)$, there exists a change of coordinates of the form $\left[\begin{array}{c}\chi_{i} \\ z_{i}\end{array}\right]=T_{i} x_{i}$, such that each system (4) can be rewritten as follows:

$$
\left[\begin{array}{c}
\dot{\chi}_{i} \\
\dot{z}_{i}
\end{array}\right]=\left[\begin{array}{cc}
A_{11 i} & A_{12 i} \\
A_{21 i} & A_{22 i}
\end{array}\right]\left[\begin{array}{c}
\chi_{i} \\
z_{i}
\end{array}\right]+\left[\begin{array}{l}
0 \\
B_{2 i}
\end{array}\right] u_{c i}
$$

where $\chi_{i} \in \mathbb{R}^{n_{i}-m}$ and

$$
\left[\begin{array}{ll}
A_{11 i} & A_{12 i} \\
A_{21 i} & A_{22 i}
\end{array}\right]=T_{i} A_{i} T_{i}^{-1} .
$$

Note that, in this representation, $A_{11 i} \in \mathbb{R}^{\left(n_{i}-m\right) \times\left(n_{i}-m\right)}$ is Hurwitz, $B_{2 i} \in \mathbb{R}^{m \times m}$ is invertible and satisfies $T_{i} B_{i}=\left[\begin{array}{ll}0 & B_{2 i}^{T}\end{array}\right]^{T}$. In Edwards and Spurgeon $(1995,1998)$, a method is proposed to construct the matrix $T_{i}$ representing the change of coordinates. The system is now rewritten in an appropriate representation to analyze the problem of measurement variable consensus. Due to previous transformation, the control law (10) can be rewritten as:

$$
u_{i}=-K_{i} x_{i}+B_{2 i}^{-1}\left[\dot{v}_{i}-\beta\left(z_{i}-v_{i}\right)-\left[0 I_{m}\right] T_{i} A_{i} x_{i}\right]
$$

Consider system (1) with the control law (12). For all $i \in \mathscr{N}$, each closed-loop system becomes:

$$
\dot{x}_{i}=\left(\bar{A}_{i}-B_{i} K_{i}\right) x_{i}+B_{i}\left(B_{2 i}\right)^{-1}\left[\dot{v}_{i}-\beta\left(z_{i}-v_{i}\right)-\left[0 I_{m}\right] T_{i} A_{i} x_{i}\right] .
$$

Following the line presented in the control design section, some computations show that the previous system is equivalent to:

$$
\left[\begin{array}{c}
\dot{\chi}_{i} \\
\dot{z}_{i}
\end{array}\right]=\left[\begin{array}{ll}
A_{11 i} & A_{12 i} \\
0 & -\beta I_{m}
\end{array}\right]\left[\begin{array}{c}
\chi_{i} \\
z_{i}
\end{array}\right]+\left[\begin{array}{l}
0 \\
\beta I_{m}-\left(L_{i}\right) \otimes I_{m} v
\end{array}\right] .
$$

Recalling that $\varepsilon=z_{i}-v_{i}$, one has:

$$
\left[\begin{array}{c}
\dot{\chi}_{i} \\
\dot{\varepsilon}_{i}
\end{array}\right]=\left[\begin{array}{ll}
A_{11 i} & A_{12 i} \\
0 & -\beta I_{m}
\end{array}\right]\left[\begin{array}{l}
\chi_{i} \\
\varepsilon_{i}
\end{array}\right]+\left[\begin{array}{l}
A_{12 i} v_{i} \\
0
\end{array}\right]
$$

On the other hand, the variable $v_{i}$ is obtained by solving a consensus problem summarized as $\dot{v}=-L \otimes I_{m} v$ which is known to be stable provided that the communication graph contains a directed spanning tree. Consequently, $\lim _{t \rightarrow+\infty}\left(v_{i}-\right.$ $\left.v_{j}\right)=0$, for all $(i, j) \in \mathscr{N}^{2}$. Finally, applying a separation principle allows concluding on the stability of the multi-agent 


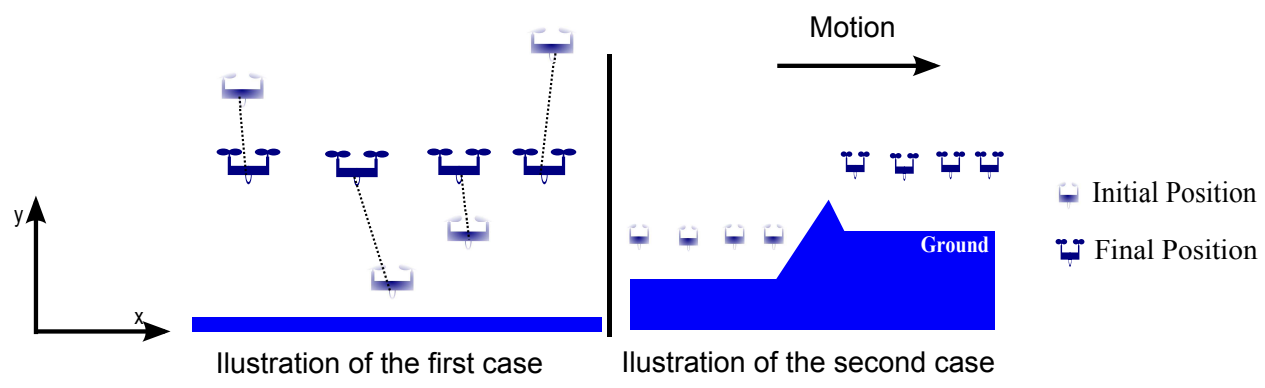

Fig. 2. Application framework

system (13) for all $i \in \mathscr{N}$. Thus, for all $i \in \mathscr{N}, \lim _{t \rightarrow+\infty}\left(v_{i}-\right.$ $\left.z_{i}\right)=0$.

It is important to point out that the method proposed in this paper allows splitting the analysis of each agent and the analysis of the distributed consensus algorithm. Therefore, it is possible to extend the previous control law to more general situations, where for instance the communication link induces transmission delays as provided in Munz et al. (2008), Seuret et al. (2008) or when one considers distributed filters as in OlfatiSaber and Shamma (2005). In fact, practical applications such as the control of a fleet of mobile robots naturally ask for environment constrained controllers or robustness to transmission delays induced by communication network. This will be detailed in the sequel.

Remark 2. An other important issue concerns the agreement point. It has been shown (see the proof of Theorem 1) that (1) will achieve consensus where $z(\infty)$ depends on the initial conditions of the consensus variables $v_{i}$. Thus, no matter which the initial state of the system is, (1) will always achieve consensus on the agreement value of $v$ (see for instance Figure 3(b)).

\section{EXTENSIONS TO MORE COMPLEX SITUATIONS}

\subsection{Consensus algorithms with transmission delays}

In this section, it is assumed that agent $i$ has access to its own variable without any delay, but receives the information from its neighbors after a time-delay caused by the communication network. Consider further as an approximation that all the communication delays are constant and equal to $\tau$ which can be assimilated as an average delay. In this situation, the additional dynamics becomes:

$$
\dot{v}_{i}(t)=\sum_{j \in \mathscr{N}_{i}} a_{i j}\left(v_{j}(t-\tau)-v_{i}(t)\right), \quad i \in\{1, \ldots, N\}
$$

where it is assumed that $\sum_{j \in \mathscr{N}_{i}} a_{i j}=1$, for all $i \in \mathscr{N}$. Knowing that the vector $\overrightarrow{\mathbf{1}}$ is an eigenvector associated to the eigenvalue "0" of the Laplacian matrix, it is possible to find a change of coordinates such that $v=W \eta$ and:

$$
W^{-1} L W=\left[\begin{array}{cc}
B & 0 \\
0^{T} & 0
\end{array}\right]
$$

where $W$ is a non singular matrix such that $W^{-1}=\left[\begin{array}{l}U_{1} \\ U_{2}\end{array}\right]$ and $U_{2}=(U)_{N}$. A stability condition of the consensus algorithm with communication delays is described as follows:

Theorem 2. [Seuret et al. (2008)] Consider the system (14) with a constant delay $\tau$. If there exists $\bar{\tau} \geq \tau$ such that:

$$
\text { - } 1+\lambda_{i}(-B-I) \frac{1-e^{s \bar{\tau}}}{s} \neq 0 \text {, for all } s \in C^{+} \text {, and }
$$

- there exist symmetric and positive-definite matrices $P, S$ such that the following LMI holds:

$$
\left[\begin{array}{cc}
B^{T} P+P B+\bar{\tau} S & B^{T} P(B+I) \\
(B+I)^{T} P B & -\bar{\tau} S
\end{array}\right]<0,
$$

then all elements of $v$ converge asymptotically to a common value $v_{e q}$ which is given by:

$$
v_{e q}=U_{2}\left(\lim _{s \rightarrow 0} s \frac{v(0)+e^{-\tau s} \int_{-\tau}^{0} v(t) e^{-t s} \mathrm{~d} t}{s+\left(1-e^{-\tau s}\right)}\right) \overrightarrow{\mathbf{1}} .
$$

Consequently, the following result holds:

Corollary 1. If Assumptions 1-5 and the conditions of Theorem 2 are satisfied, the control law (10) where $\dot{v}$ is given in (14), ensures that the multi-agent system (1) is stable and reaches an asymptotic measurement variable consensus.

\subsection{Consensus algorithms with external reference}

In this section we focus on reference-based consensus algorithms (see Olfati-Saber and Shamma (2005)). Our objective here is to develop a distributed algorithm that allows the agents to track an external signal while the consensus agreement is achieved. It is considered that each agent receives a different signal, or the same signal corrupted by noise for instance. In this case the objective is to achieve a measurement variable consensus while tracking the average of all external signal references. Let $r_{i} \in \mathbb{R}^{m}$ be the external signal reference received by agent $i \in \mathscr{N}$. In this situation, the new additional system can then be expressed as

$$
\dot{v}_{i}=-\alpha \sum_{j \mathscr{N}_{i}}\left(v_{i}-v_{j}\right)+\sum_{j \in \mathscr{J}_{i}}\left(r_{j}-v_{i}\right)
$$

where $\mathscr{J}_{i}=\mathscr{N}_{i} \cup\{i\}$ and $r=\left[r_{1}, \ldots, r_{N}\right]^{T}$ denotes the external signals. As mentioned before, our objective is to reach an agreement on the average of measurements such that $v_{i} \rightarrow$ $r_{c}, \forall i \in \mathscr{N}$ where $r_{c}=\frac{1}{N} \sum_{i=1}^{N} r_{i}$.

Theorem 3. [Olfati-Saber and Shamma (2005)] Let $r_{i}$ be a signal with uniformly bounded rate such that following inequality is satisfied $\left\|r^{*}\right\| \leq \gamma_{1}$, where $r^{*}=\overrightarrow{\mathbf{1}} \otimes r_{c}$. Then $v=r^{*}$ is a globally asymptotically $\varepsilon$-stable equilibrium of the consensus algorithm given by (17) with

$$
\varepsilon=\frac{\left.\gamma_{1} \sqrt{N}\left(1+d_{\max }\right)+\gamma_{2} \gamma_{3}\right) \lambda_{\max }^{1 / 2}\left(A_{\alpha}\right)}{\lambda_{\min }^{5 / 2}\left(A_{\alpha}\right)}
$$

where constants $\gamma_{2}$ and $\gamma_{3}$ are defined as

$$
\left\|r-r^{*}\right\| \leq \gamma_{2}, \quad\left\|B_{\alpha}^{T} A_{\alpha}\right\| \leq \gamma_{3},
$$

and where $A_{\alpha}=\alpha L+I_{N}+\Delta$ and $B_{\alpha}=I_{N}+\mathscr{A}$.

In our case, the following result holds: 


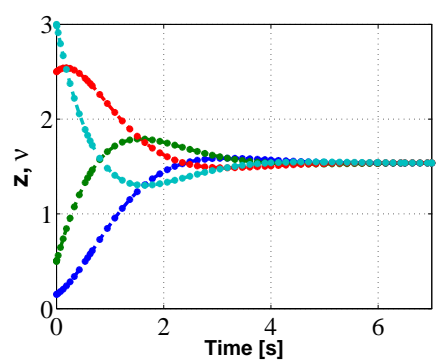

(a) Evolution of the variables $v_{i}$ 's and measurements $z_{i}$ 's when $v_{i}(0)=z_{i}(0)$

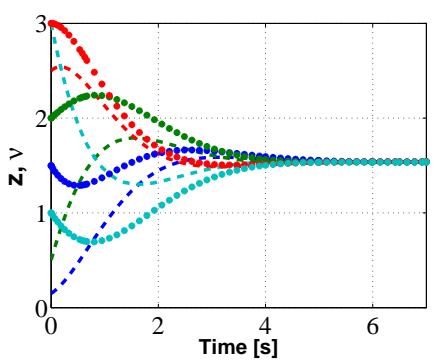

(b) Evolution of the variables $v_{i}$ 's and mea- (c) Evolution of the delayed variables $v_{i}$ 's surements $z_{i}$ 's when $v_{i}(0) \neq z_{i}(0)$

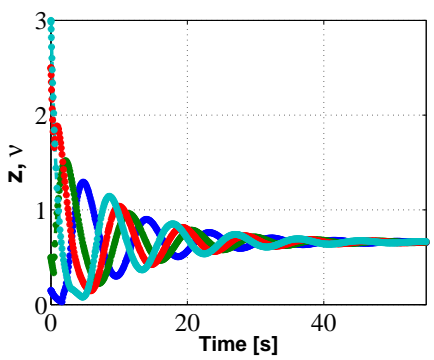

$(\tau=0.6)$ and measurements $z_{i}$ 's, when $v_{i}(0)=z_{i}(0)$

Fig. 3. Evolution of the additional dynamics $v$ (dashed line) and the measurement vector $z$ (star line).

Corollary 2. If Assumptions 1-5 and the conditions of Theorem 3 are satisfied, the control law (10) where $\dot{v}$ is given in (17), ensures that the multi-agent system (1) is stable and reaches an measurement variable $\varepsilon$-consensus.

\section{EXAMPLES AND SIMULATION RESULTS}

In this section, some results regarding the efficiency of the approach introduced in the previous sections will be presented. We are particularly interested in applications considering the motion control of a set of mobiles robots. Therefore, consider a set of $N=4$ heterogeneous flying robots (Figure 2). In order to fit with our problem, the matrices $\bar{A}_{i}, B_{i}$, and $C_{i}$ for the different agents are defined by

$$
\begin{aligned}
& \bar{A}_{1}=\left[\begin{array}{cc}
-1 & 0.5 \\
0.05 & -1
\end{array}\right], \quad B_{1}=\left[\begin{array}{l}
0 \\
1
\end{array}\right], \quad C_{1}=\left[\begin{array}{l}
0 \\
1
\end{array}\right]^{T}, \\
& \bar{A}_{2}=\left[\begin{array}{cc}
-2 & 1 \\
0 & -0.9
\end{array}\right], \quad B_{2}=\left[\begin{array}{l}
-1 \\
-1
\end{array}\right], C_{2}=\left[\begin{array}{l}
0 \\
1
\end{array}\right]^{T}, \\
& \bar{A}_{3}=\left[\begin{array}{ccc}
0 & 1 & 1 \\
-2 & 0.1 & 2 \\
1 & 2 & 3
\end{array}\right], \quad B_{3}=\left[\begin{array}{l}
0 \\
1 \\
1
\end{array}\right], \quad C_{3}=\left[\begin{array}{l}
0 \\
0 \\
1
\end{array}\right]^{T}, \\
& \bar{A}_{4}=\left[\begin{array}{cccc}
0 & 1 & 1 & 0 \\
-2 & 0.1 & 2 & 2 \\
0 & 1 & 2 & 3 \\
0.2 & 0.4 & 0.1 & 0.3
\end{array}\right], B_{4}=\left[\begin{array}{l}
1 \\
0 \\
1 \\
1
\end{array}\right], \quad C_{4}=\left[\begin{array}{l}
0 \\
0 \\
1 \\
0
\end{array}\right]^{T}
\end{aligned}
$$

It is clear that the agents are characterized by different dimensions and stability properties. Moreover Assumptions 1-4 are fulfilled. Thus, a pole placement allows us to find matrices $K_{i}$ 's such that the matrices $A_{i}=\bar{A}_{i}-B_{i} K_{i}$ are Hurwitz.

Consider that these four agents are connected trough a graph expressed by the following Laplacian matrix

$$
L=\left[\begin{array}{cccc}
1 & -1 & 0 & 0 \\
0 & 1 & -1 & 0 \\
0 & 0 & 1 & -1 \\
-1 & 0 & 0 & 1
\end{array}\right]
$$

Note that this choice is not restrictive, as long as $L$ represents a graph containing a spanning tree satisfying Assumption 5.

In a first case, the heterogeneous agents must agree on the same height by applying the control strategy detailed in Section 3. Related to Remark 2, a special attention is paid to the influence of non-identical initial conditions (between the additional model and the multi-agent system) over convergence properties. In a second case, the robot fleet is moving trough a changing environment. More precisely, the ground profile varies with the position of the fleet, while the objective aims to keep a constant height for the fleet with respect to the ground. This particular application has a pertinent practical meaning
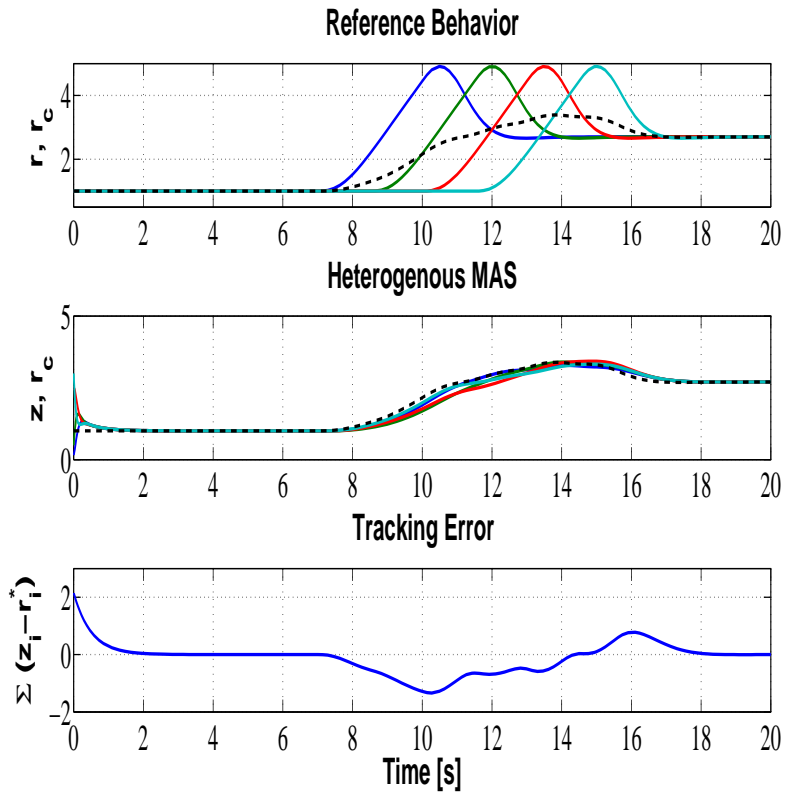

Fig. 4. Reference signals and heterogeneous MAS response

since, in several operations, such as search and recovery operations, robots usually move trough changing/hostile environment. Therefore, distributed collaboration, by exchanging individual measurements in order to achieve the control objectives, becomes a key issue.

Consider for the moment Figure $3^{1}$. Figure 3(a) shows simulation results for the closed-loop system (1) controlled by (2). We can clearly see that both systems reach a consensus, where the agreement value corresponds to $v(\infty)$. Note that, since the chosen $L$ matrix is doubly stochastic, $v(\infty)$ corresponds to the average of the initial conditions of the additional model, i.e, $v(\infty)=$ ave $\{v(0)\}$. Figure $3($ b) shows the same set of heterogeneous agents initialized with different initial conditions from those of the additional variables. Once more, we can see that system (1) achieve consensus, converging to the agreement value of the additional model $v(\infty)$. This simulations enhance the conclusion mentioned in Remark 2, as well as the advantages of the referenced based algorithm introduced in Section 5.2. In Figure 3(c), we can find simulation results for the closedloop system (1) controlled by the delayed additional algorithm

\footnotetext{
1 For all figures, the dashed line corresponds to the additional dynamics, whereas the star line represents the measurement vector $z$ evolution.
} 
(14). For these simulations, we considered that all the communication delays are constant and equal to $\tau=0.6 s$, which in other words can be assimilated as an average delay. Note that in Figure 3(a),3(c) both the dashed line and the star line are completely overlapped due to equal initial conditions. Figure 3(c) shows that all elements of both the delayed additional dynamics $v_{i}$ and the measurement vector $z_{i}$, asymptotically converge to a common value $v_{e q}$ previously defined. These results enhance the efficiency of the proposed strategy when more complex situations are considered for the additional system.

Consider now the application setup illustrated in Figure 2. While previous simulation results concern the first case, where flying robots must agree on a common height, consider now the second application case assuming that agents are moving on a changeable environment. Initially, agents are supposed to agree on a same height. At a certain time instant different for each agent $i$, the ground level changes due to a cliff or an obstacle. Therefore, such a case naturally asks for the external referencebased control strategy mentioned in Section 5.2. Thus, agents are supposed to perform an efficient reference tracking and to finally agree on a common height. Figure $4^{2}$ shows the simulation of four flying vehicles controlled by the reference based control law (17), where the input signals $r_{i}$ are different for every agent $i$. We can see the evolution of the measurement vector $z$ of system (1), as well as the evolution of the error $z_{i}-r_{c}$, where $r_{c}$ corresponds to the average of the reference signals. Therefore, we can see that all measurements vectors $z_{i}$ of system (1) on a common value $r_{c}$, satisfying our control objectives. All previous results show the efficiency of our approach. We have shown that by using simple additional dynamics, the control of a high order heterogeneous multiagent system not only becomes possible, but can be done with low constraints on system (1) and with low calculation load.

\section{CONCLUSIONS}

In this paper, a novel approach for heterogeneous multi-agent systems to reach measurement variable consensus has been presented, designed and analyzed. The major advantage of the proposed algorithm remains in the separation of the stability analysis of each subsystem and the distributed control algorithm. It has been shown that the method allows using classical distributed consensus algorithm for simple integrator dynamics in order to derive a appropriated control law for a complex system composed of non-identical agents. Furthermore, we have also shown that different additional dynamics can be used, such as delayed simple integrator consensus and distributed consensus filter algorithms. This approach, when compared with the available results on literature, appears to have good performances, since other solutions offer some drawbacks as calculation complexity or restrictive assumptions.

\section{REFERENCES}

N. Chopra and W. Spong. Output synchronization of nonlinear systems with relative degree one. Recent. Adv. Learning Control, LNCIS371:51-64, 2008.

D. V. Dimarogonas and K. J. Kyriakopoulos. A connection between formation infeasibility and velocity alignment in kinematic multi-agent systems. Automatica, 44:2648-2654, 2008 .
C. Edwards and S. K. Spurgeon. Sliding mode stabilization of uncertain systems using only output information. International Journal of Control, 62(5):1129-1144, 1995.

C. Edwards and S. K. Spurgeon. Sliding Mode Control: Theory and Applications. London, U.K.: Taylor and Francis, 1998.

J.P. Hespanha, P. Naghshtabrizi, and Y. Xu. A survey of recent results in networked control systems. Proceedings of IEEE, 95(1):138 - 162, 2007

A. Isidori. Nonlinear Control Systems. Springer, 1995.

U.T. Jönsson and C.-Y. Kao. Consensus of heterogeneous linear agents applied to a formation control problem. In $49^{\text {th }}$ IEEE Conference on Decision and Control, 2010.

H. Kim, H. Shim, and J. H. Seo. Output consensus of heterogeneous uncertain linear multi-agent systems. IEEE Transactions on Automatic Control, 56(1):200-206, 2011.

U. Munz, A. Papachristodoulou, and F. Allgöwer. Delaydependent rendezvous and flocking of large scale multiagent systems with communication delays. In $47^{\text {th }}$ IEEE Conference on Decision and Control, 2008.

P. Ögren, E. Fiorelli, and N. E. Leonard. Cooperative control of mobile sensor networks: Adaptive gradient climbing in a distributed environment. IEEE Transactions on Automatic Control, 49:1292-1302, 2004.

R. Olfati-Saber and R. M. Murray. Consensus problems in networks of agents whith switching topology and time-delays. IEEE Transactions on Automatic Control, 49(9):1520-1533, 2004.

R. Olfati-Saber and J. S. Shamma. Consensus filters for sensor networks and distributed sensor fusion. In $44^{\text {th }}$ IEEE Conference on Decision and Control, 2005.

R. Olfati-Saber, J.A. Fax, and R.M. Murray. Consensus and cooperation in networked multi-agent systems. Proceedings of the IEEE, 95(1):215 -233, 2007.

Z. Qu, J. Chunyu, and J. Wang. Nonlinear cooperative control for consensus of non linear and heterogeneous systems. In IEEE Conference on Decision and Control, 2007.

W. Ren. Consensus strategies for cooperative control of vehicle formations. IET Control Theory \& Applications, 1(2):505$512,2007$.

W. Ren and R. W. Beard. Distributed Consensus in Multivehicle Cooperative Control. Communication and Control Engineering. Springer, 2007.

W. Ren, R. W. Beard, and E. M. Atkins. A survey of consensus problems in multi-agent coordination. In American Control Conference, 2005.

L. Scardovi and R. Sepulchre. Synchronization in networks of identical linear systems. Automatica, 44(11):2557-2562, 2009.

A. Seuret, D. V. Dimarogonas, and K. H. Johansson. Consensus under communication delays. In $47^{\text {th }}$ IEEE Conference on Decision and Control, 2008.

P. Wieland, J.-S. Kimb, and F. Allgöwer. On topology and dynamics of consensus among linear high-order agents. International Journal of Systems Science, 42, 2011a.

P. Wieland, R. Sepulchre, and F. Allgöwer. An internal model principle is necessary and sufficient for linear output synchronization. Automatica, 47:1068-1074, $2011 \mathrm{~b}$.

L. Xiao and S. Boyd. Faster linear iterations for distributed averaging. Systems \& Control Letters, 53:65-78, 2004.

Y. Zheng, Y. Zhu, and L. Wang. Consensus of heterogeneous multi-agent systems. IET Control Theory and Applications, 5:1881-1888, 2011

2 For all figures, $r_{c}$ is displayed as a black dashed line. 